This document is the Accepted Manuscript version of a

Published Work that appeared in final form in Organic

Chemistry Frontiers, copyright (C) Royal Society of

Chemistry, after peer review and technical editing by the publisher.

To access the final edited and published work see

Organic Chemistry Frontiers 2021, 8, 549-554

https://doi-org.uml.idm.oclc.org/10.1039/D0QO01311K

Also see same web-link for Supporting Information, available free of charge. 


\title{
ARTICLE
}

\section{Electrochemical Hydrogenation of $\alpha$-Ketoesters and Benzoxazinones Using Carbon Electrodes and a Sustainable Brønsted Acid}

Received 00th January 20xx Accepted 00th January 20xx DOI: $10.1039 / \times 0 \times x 00000 x$

\author{
Dion B. Nemez ${ }^{\mathrm{a}}$, Baldeep K. Sidhu ${ }^{\mathrm{a}}$, Patrick K. Giesbrecht ${ }^{\mathrm{a}}$, Jason D. Braun, ${ }^{\mathrm{a}}$ and David E. Herbert*a
}

\begin{abstract}
The direct electrochemical hydrogenation of unsaturated organic compounds containing $\mathrm{C}=\mathrm{N}$ and $\mathrm{C}=\mathrm{O}$ bonds using glassy carbon electrodes and a widely available Brønsted acid is demonstrated for a selection of $\alpha$-ketoesters and benzoxazinones. In this simple set-up, the reduced products are formed with excellent selectivity, without the use of a redox mediator, and are easily recovered in good yields by extraction. The carbon-based electrode is essential to observation of hydrogenated product; no turnover is observed using platinum mesh.
\end{abstract}

\section{Introduction}

Electrochemical synthesis is a promising approach to the preparation of organic fuels, synthons and complex molecules, ${ }^{1,2}$ that can exploit renewables-sourced electricity on the laboratory ${ }^{3}$ or industrial ${ }^{4}$ scale. Direct, uncatalyzed electrochemical hydrogenation ${ }^{5}$ is further interesting from a sustainability perspective ${ }^{6}$ as petroleum-derived $\mathrm{H}_{2}$ can be replaced with renewable Brønsted acids ${ }^{7}$ and the reaction avoids the use of added redox mediators. Ideally, in such processes, abundant electrode materials would be employed; however, examples using nickel $^{8}$ and iron electrodes ${ }^{9,10}$ can suffer from low efficiencies. As a result, precious metals (e.g., $\mathrm{Pd}, \mathrm{Pt}$ ) have been more often utilized to improve performance. ${ }^{11}$

Planar glassy carbon (GCE) and mesh-like reticulated vitreous carbon (RVC) electrodes (which provide higher surface area but are otherwise chemically identical to GCEs) are appealing cost-effective alternatives made from abundant materials $^{12}$ and their inertness may be advantageous in achieving product selectivity and limiting overreactions. ${ }^{7}$ Despite these potential advantages, carbon electrodes have been most commonly employed as supports for electrochemically active films ${ }^{13,14}$ or catalysts. ${ }^{15}$ Their application in direct, electrochemical modifications of organic materials has been comparatively limited. ${ }^{16-21}$ Here, we report that unsaturated $\alpha$-ketoester and benzoxazinone substrates can be selectively hydrogenated at carbon electrodes using sustainable Brønsted acids such as acetic acid (Figure 1).

\footnotetext{
a. Department of Chemistry

University of Manitoba

144 Dysart Road, Winnipeg, Manitoba, CANADA

*david.herbert@umanitoba.ca

† Electronic Supplementary Information (ESI) available: full experimental details including electrochemical methodology, CPE plots, cyclic voltammograms, multinuclear NMR spectra, control experiments and details of $\mathrm{X}$-ray analysis; crystallographic information files. See DOI: 10.1039/x0xx00000x
}

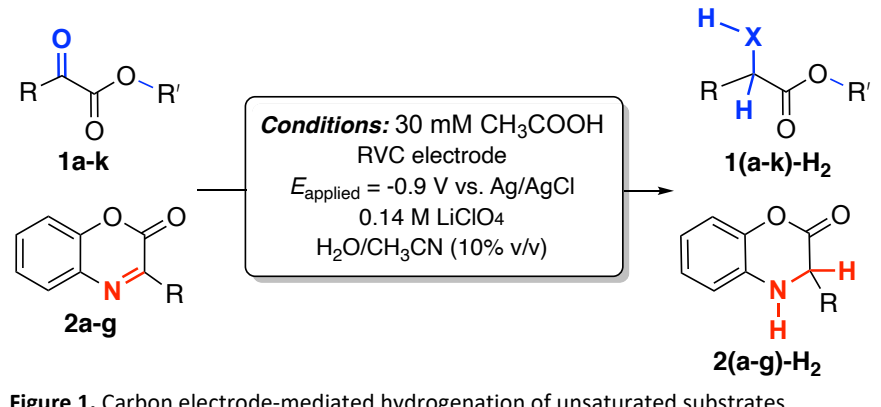

\section{Results and discussion}

The complete hydrogenation of pyridinium to piperidine via controlled-potential electrolysis using $\mathrm{Pt}$ electrodes under suitably acidic conditions has been reported, ${ }^{22}$ with surface proton transfer implicated in the reactivity. ${ }^{23}$ These observations corroborate computational studies that suggest coupled $2 \mathrm{e}^{-} / 2 \mathrm{H}^{+}$reductions of $\mathrm{N}$-heterocycles are thermodynamically accessible in the range of the reported applied potentials. ${ }^{24,25}$ Similarly, the selective hydrogenation of benzannulated pyridines such as phenanthridine to form 1,2dihydrophenanthridine has also been observed when the equivalents of acid present are appropriately controlled. ${ }^{26}$ To probe the generality of this process, we sought to investigate the electrochemical hydrogenation of two substrate classes, $\alpha$ ketoesters and benzoxazinones (Figure 2), that have been previously explored in the context of biomimetic dihydrophenanthridine-mediated transfer hydrogenation. ${ }^{27,28}$

First, cyclic voltammograms (CVs) of $\alpha$-ketoester (1a-k) and benzoxazinone (2a-g) substrates were recorded using freshly polished glassy carbon disc electrodes (surface area $=7.068$ $\mathrm{mm}^{2}$ ) under neutral and acidic conditions. Representative CVs of ethyl 2-oxo-2-(4-chlorophenyl)acetate (1a) and 3-(4chlorophenyl)-2H-1,4-benzoxazin-2-one (2a) are shown in Figure 3 . In acetonitrile, the first accessible reductions observed 
for $\mathbf{1 a - k}$ are generally quasi-reversible (e.g., $1 \mathrm{a}: E_{1 / 2}=-1.66 \mathrm{~V}$ vs. $\mathrm{FcH}^{0 /+}, i_{\mathrm{a}} / i_{\mathrm{c}}=0.50 ; \mathrm{FcH}=$ ferrocene). In comparison, reduction of the cyclic benzoxazinones $\mathbf{2 a - g}$ are largely more fully reversible (e.g., $2 \mathrm{~d}: E_{1 / 2}=-1.59 \mathrm{~V}$ vs. $\mathrm{FcH}^{\circ /+}, i_{\mathrm{a}} / i_{\mathrm{c}}=0.98$ ), with the exception of $2 \mathrm{~g}\left(E_{1 / 2}=-1.46 \mathrm{~V}\right.$ vs. $\left.\mathrm{FcH}^{0 /+}, i_{\mathrm{a}} / i_{\mathrm{c}}=0.48\right)$. Ketoester 1a shows two additional quasi-reversible events $\left(E_{1 / 2}=-2.58\right.$ and $-2.77 \mathrm{~V}$ vs. $\mathrm{FCH}^{0 /+}$ ) within the solvent window, while a second event of diminished current response is seen for $2 \mathrm{a}\left(E_{1 / 2}=-2.15 \mathrm{~V}\right.$ vs. $\mathrm{FcH}^{0 /+}$ ). CVs for all compounds are shown in the Supporting Information (Figures S1-S18) and summarized in Table S1.

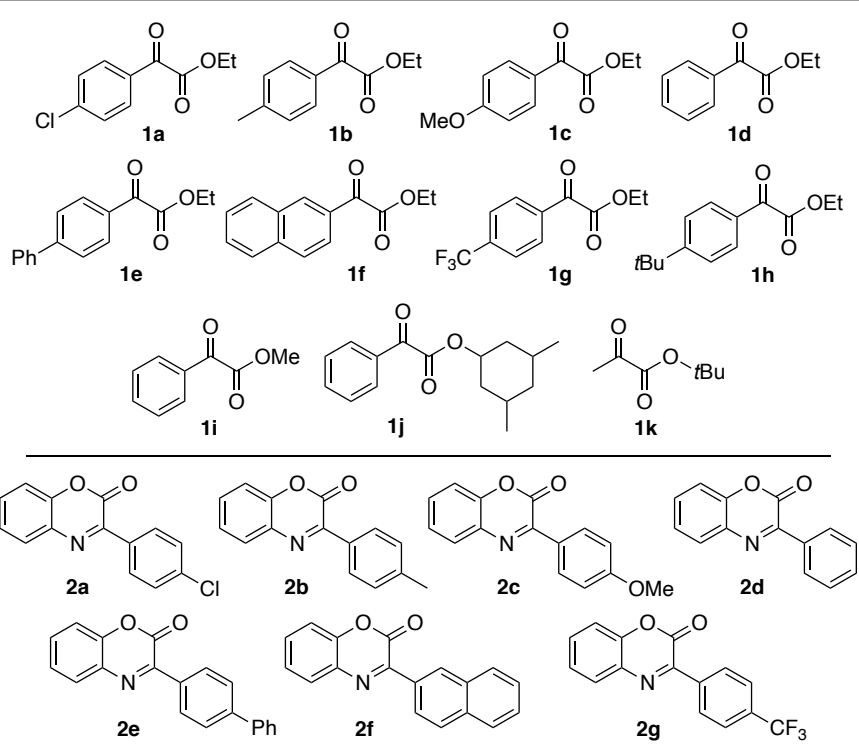

Figure 2. Substrate scope.

In the presence of added aqueous acetic acid (AA), the primary cathodic events for all substrates shift to more positive potentials. In general, the voltammograms of $\mathbf{2 a - g}$ were more responsive to a decrease in $\mathrm{pH}(\Delta E=0.16-0.42 \mathrm{~V})$ compared with 1a-k ( $\Delta E=0.01-0.24 \mathrm{~V})$, consistent with the higher basicity of the benzoxazinone nitrogens reflecting a larger equilibrium concentration of protonated substrate. In both cases, the reductive events are fully irreversible in the presence of exogenous acid. The electrochemistry of ketones is similarly responsive to the presence of protic solvents, an observation that has been attributed both to hydrogen bonding and the onset of coupled proton-electron transfer. ${ }^{13}$ Here, the different shifts in the reduction peaks of the substrates in the presence of added $A A$ and the onset of irreversibility likewise implies reduction of protonated substrate and subsequent coupled proton-electron transfer reactivity. Alternatively, a 'weak-acid' mechanism ${ }^{29}$ mediated by the substrate may be operative as shown for Pt electrodes, forming surface-adsorbed hydrogens $\left(\mathrm{H}_{\text {ads }}\right)$, though this should be more challenging for glassy carbon; the position of the new redox events are nevertheless overall inconsistent with the electrochemical reduction of acetic acid alone. ${ }^{30}$ Given the possibility of coupled proton-electron transfer and/or formation of $\mathrm{H}_{\mathrm{ads}}$, we hypothesized that over longer timescales, substrate hydrogenation might be accessible and outcompete dimerization pathways, as observed with the selective electrochemical hydrogenation of phenanthridine. ${ }^{26}$

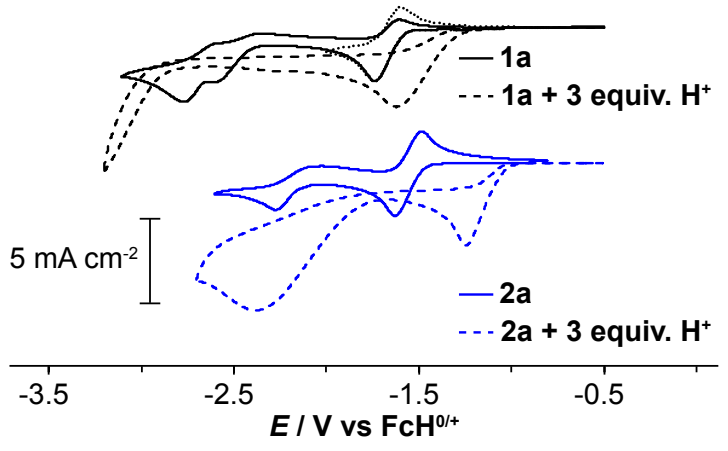

Figure 3. Cyclic voltammograms of $10 \mathrm{mM}$ 1a and $2 \mathrm{a}$ in $\mathrm{CH}_{3} \mathrm{CN}$ with $0.1 \mathrm{M}$ $n \mathrm{Bu}_{4} \mathrm{NPF}_{6}$ (solid traces) and in the presence of $30 \mathrm{mM} \mathrm{CH}_{3} \mathrm{COOH}$ (dashed lines). Conditions: Glassy carbon disk working electrode, $\mathrm{Ag} / \mathrm{AgCl}$ reference electrode, $\mathrm{Pt}$ wire counter electrode, scan rate $=100 \mathrm{mVs}^{-1}$.

Controlled potential electrolyses (CPE; see Figure S19) ${ }^{31}$ of substrates 1a-j and 2a-g (1 mM) were therefore carried out in the presence of three equivalents of $A A$ as a proton source ( 3 $\mathrm{mM})$, at a set applied potential of $-0.9 \mathrm{~V} \mathrm{vs}$. $\mathrm{Ag} / \mathrm{AgCl}(-1.15 \mathrm{~V}$ vs. $\left.\mathrm{FcH}^{0 /+}\right)$. A mixed solvent medium $\left(10 \% \mathrm{v} / \mathrm{v}_{2} \mathrm{O} / \mathrm{CH}_{3} \mathrm{CN}\right)$ was used based on previous findings of the importance of water in facilitating electrochemical hydrogenation of benzannulated pyridines. ${ }^{26}$ Indeed, lower conversion was observed for the hydrogenation of $\mathbf{1 d}$ in neat acetonitrile, with a lower average current consumption in the purely organic medium likely also reflecting the change in reaction medium conductivity (Table S2). Once the rate of charge passed had slowed, the experiment was ended. Following simple solvent extraction to separate the organic material from the electrolyte, ${ }^{1} \mathrm{H}$ NMR spectroscopy revealed the corresponding hydrogenated species [i.e., 1(a-j)$\mathbf{H}_{\mathbf{2}}$ and $\mathbf{2}(\mathrm{a}-\mathrm{g})-\mathbf{H}_{\mathbf{2}}$ ] were generated quite selectively (>90\% conversion), with no evidence of dimers, hydrodimers or other side products. Figure 4 shows ${ }^{1} \mathrm{H}$ NMR of thus-isolated $\mathbf{1} \mathbf{b}-\mathbf{H}_{\mathbf{2}}$ stacked with that of the oxidized starting material $\mathbf{1 b}$. In the former spectrum, only two compounds are visible: the major product, hydrogenated $\mathbf{1} \mathbf{b}-\mathbf{H}_{\mathbf{2}}(\mathbf{9 7 \%})$, and a small amount of unreacted starting material $\mathbf{1 b}(\mathbf{b} \%)$. The product can clearly be identified by the appearance of two additional signals integrating to $1 \mathrm{H}$ each, including a sharp singlet $(\mathrm{CH})$ corresponding to the hydrogenated product ( $\delta=5.5 \mathrm{ppm}$ ).

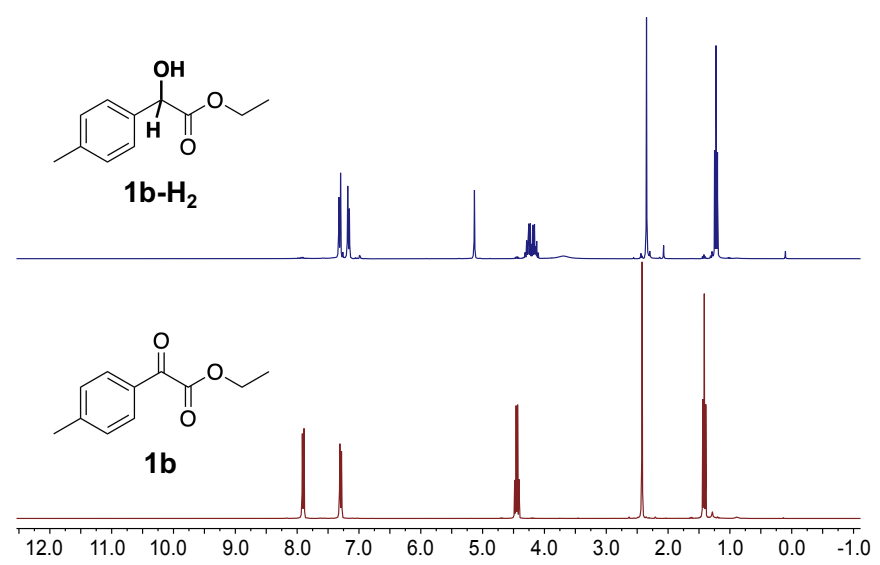

Figure 4. ${ }^{1} \mathrm{H}$ NMR spectra $\left(300 \mathrm{MHz}, \mathrm{CDCl}_{3}, 22^{\circ} \mathrm{C}\right.$ ) of $\mathbf{1 b}$ and electrochemically hydrogenated $\mathbf{1} \mathbf{b}-\mathbf{H}_{\mathbf{2}}$ isolated from electrolysis cell without further purification. 
To confirm the chemoselectivity of hydrogenation assigned by multi-nuclear NMR spectroscopy, both $\mathbf{2 g}$ and its reduced product $\left(\mathbf{2} \mathbf{g}-\mathbf{H}_{2}\right)$ were characterized in the solid-state using X-ray diffraction (Figure 5). Compared with $\mathbf{2 g}$, the N1-C7 bond length is longer in $\mathbf{2 g}-\mathbf{H}_{\mathbf{2}}(1.457 \AA$; cf. $\mathbf{2 g} 1.296 \AA$ ). Additionally, the $\mathrm{N} 1 / \mathrm{C} 7 / \mathrm{C} 8$ bond angle has decreased from $122.00^{\circ}$ to $109.02^{\circ}$, as expected from formation of a tetrahedral $s p^{3}$ carbon. $\mathbf{2 g}-\mathbf{H}_{\mathbf{2}}$ crystallized in a non-centrosymmetric space group $C c$ with a Flack parameter of 0 , indicating an enantiopure crystal. Analysis of the bulk mixture via chiral column supercritical fluid ultrahigh-performance liquid chromatography (SFC-UHPLC), however, indicated the overall product was a racemate.
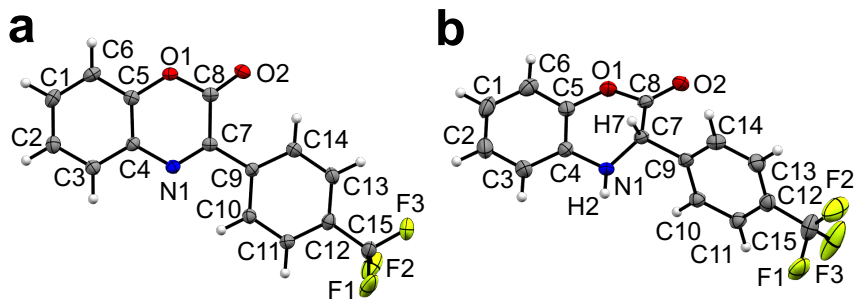

Figure 5. Solid-state structures of (a) $\mathbf{2 g}$ and (b) $\mathbf{2} \mathbf{g}-\mathrm{H}_{2}$ with selected atom labels omitted for clarity. Thermal ellipsoids are shown at $50 \%$ probability. Selected bond distances ( $(\AA)$ and angles $\left({ }^{\circ}\right)$ for $2 \mathrm{~g}$ : N(1)-C(7) 1.296(2), N(1)-C(4) 1.390(2), C(7)-C(8) 1.497(2), C(7)-C(9) 1.490(2); C(4)-N(1)-C(7) 119.19(14), N(1)-C(7)-C(8) 122.00(15), $\mathrm{N}(1)-\mathrm{C}(7)-\mathrm{C}(9)$ 117.80(15), C(9)-C(7)-C(8) 120.20(14). 2g-H : N(1)-C(7) 1.457(4), $N(1)-C(4) 1.398(4), C(7)-C(8) 1.515(4), C(7)-C(9) 1.511(4) ; C(4)-N(1)-C(7) 114.5(2)$, $N(1)-C(7)-C(8) 109.0(2), N(1)-C(7)-C(9) 111.6(2), C(9)-C(7)-C(8) 111.2(2)$.

Electrolyses at higher concentrations (3-6 mM) enabled isolation and complete characterization of the hydrogenated products (Table 1). The CPE plots were found to level off after ca. 2 electrons per equivalent of substrate (see Supporting Information). Given the excess acid utilized to perform these hydrogenations, the excess charge passed is attributed to the reduction of $A A$ in solution to hydrogen $\left(\mathrm{H}_{2}\right)$ under the potentials applied, as well as Ohmic losses in the system. Indeed, measuring the solution $\mathrm{pH}$ before and after electrolysis of $\mathbf{1 b}$, a drop in proton concentration corresponding to the excess charged passed was observed (see $\mathrm{SI}$ ). Extraction of the products from the electrolysis solution confirmed the majority species generated in each case is hydrogenated substrate (see SI for comprehensive NMR analysis). No dimers or side products were observed, with only residual starting material observed beyond hydrogenated product. Under the conditions employed, dimer formation appears not to be favourable compared with $2 \mathrm{H}^{+} / 2 \mathrm{e}^{-}$reduction of the substrates. As higher concentrations might be expected to favour bimolecular dimerization, 32 the absence of such pathways is consistent with a 'weak-acid' reduction 29,33 of the electrode followed by hydride attack on the substrates, rather than direct, $1 \mathrm{e}^{-}$reduction of the substrate. No conversion to product was observed when Pt mesh was employed in place of the RVC working electrode, highlighting the importance of electrode material choice here. ${ }^{34}$

Compounds 1 a-h present a combination of electronic and steric variances at the 4-position of the phenyl substituent, para to the ketoester moiety. Altering the electronic nature of the phenyl ring through the introduction of electron- donating/withdrawing groups resulted in a predictable shift in $E_{1 / 2}\left[\right.$ e.g., $1 \mathrm{c}-1.84(\mathrm{R}=\mathrm{OMe})$ vs. $1 \mathrm{~g}-1.60\left(\mathrm{R}=\mathrm{CF}_{3}\right) \vee$ vs. $\left.\mathrm{FcH}^{0 /+}\right]$.

\begin{tabular}{|c|c|c|c|}
\hline Substrate & $\begin{array}{c}\% \\
\text { Conversion } \\
\text { to } 1 / 2-\mathrm{H}_{2}^{[b]}\end{array}$ & $\begin{array}{c}\text { \% Mass } \\
\text { Recovery }^{[c]}\end{array}$ & $\begin{array}{c}\text { Faradaic } \\
\text { Efficiency / \% } \\
{[\mathrm{d}]}\end{array}$ \\
\hline $1 a$ & 93 & 74 & 26 \\
\hline $1 b$ & 92 & 96 & 25 \\
\hline 1c & 96 & 75 & 30 \\
\hline $1 d$ & $90^{[\mathrm{e}]}$ & $68^{[\mathrm{e}]}$ & $30^{[\mathrm{e}]}$ \\
\hline $1 d$ & $64^{[f]}$ & $72^{[f]}$ & $26^{[f]}$ \\
\hline $1 e$ & 93 & 78 & 21 \\
\hline $1 f$ & 97 & 67 & 12 \\
\hline $1 \mathrm{~g}$ & 91 & 67 & 17 \\
\hline $1 \mathrm{~h}$ & 94 & 71 & 14 \\
\hline $1 \mathbf{i}$ & 86 & 53 & 15 \\
\hline $1 \mathrm{j}$ & 99 & 99 & 49 \\
\hline $1 k$ & n.d. & - & - \\
\hline $2 a$ & 99 & 91 & 40 \\
\hline $2 b$ & 97 & 98 & 39 \\
\hline 2c & 99 & 97 & 38 \\
\hline $2 d$ & $98^{[\mathrm{e}]}$ & $99^{[\mathrm{e}]}$ & $40^{[\mathrm{e}]}$ \\
\hline $2 e$ & 98 & 95 & 36 \\
\hline $2 f$ & 96 & 89 & 36 \\
\hline $2 g$ & 87 & 99 & 32 \\
\hline
\end{tabular}

[a] Conditions: $E_{\text {app }}=-0.90$ V vs. $\mathrm{Ag} / \mathrm{AgCl}\left(-1.15 \vee v s . \mathrm{FcH}^{0 /+}\right), 10 \% \mathrm{v} / \mathrm{v} \mathrm{H}_{2} \mathrm{O} / \mathrm{CH}_{3} \mathrm{CN}$, $0.1 \mathrm{M} \mathrm{LiClO}_{4}$, reticulated vitreous carbon (RVC) working electrode $\left(\sim 700 \mathrm{~cm}^{2}\right)$, graphite rod counter electrode in a fritted tube, $\mathrm{Ag} / \mathrm{AgCl}$ quasi-reference electrode, constant sparge with $\operatorname{Ar}(1 \mathrm{~atm})$. [b] Determined through comparison of ${ }^{1} \mathrm{H}$ NMR signal integrals of hydrogenated product and unreacted starting material. [c] Represents \% mass of substrate recovered following simple solvent extraction to remove electrolyte at the level of purity indicated by the \% conversion. The remnant material in each case was unreacted starting material. [d] Determined from control potential electrolysis plots (Figures S1-S17). [e] No product was detected when Pt mesh was used as the working electrode. [f] Solvent was pure acetonitrile.

This impact, however, was not mirrored in conversion rates for the bulk hydrogenations. These were generally high, exceeding $90 \%$, with the exception of $1 \mathbf{i}$ (86\%) which also showed the smallest mass recovery by extraction. Substrate $\mathbf{1 j}$ showed the highest ( $50 \%)$ Faradaic efficiency (\%FE). Interestingly, of the ketoesters, this substrate showed one of the most reversible redox couples in the absence of added acid. Runs using substrate 1f were among the lowest in \%FE, suggesting that the substituent at this position may not play a role in facilitating favourable interactions between the substrate and the electrode surface. We note that CPE experiments were not optimized for charge passed but stopped once current consumption was observed to have levelled off (see Figures S20-S36). When monitoring conversion vs. time in the electrolysis of $\mathbf{1 a}$, an increase in conversion over time was observed (NMR analysis of reaction aliquots) and a corresponding drop in \%FE [\% conversion, \%FE (time) $=80,42$ (3 000 s); 91, 30 (6 000 s); 93, 26 (12 000 s); Fig. S37]. Thus, while near quantitative conversion of substrate to product can be obtained, a background reduction of protons contributes to current consumption over time, decreasing the \%FE of the overall process. 
While the steric bulk present on the phenyl ring had little effect on the hydrogenation process, the steric bulk of the ester correlated with bigger changes in conversion, mass recovery, and \%FE. When the steric hindrance of the ester is reduced from an ethyl (1d) to a methyl (1i), the mass recovery was lower $(53 \%$ vs. $68 \%$ ) and $\% F E$ dropped from $30 \%$ to $15 \%$. Accordingly, when the steric environment of the ester was increased from ethyl (1d) to 1,2-dimethylcyclohexyl (1j), an increase in mass recovery was observed, improving to $99 \%$ with a corresponding increase in $\mathrm{FE}$ to $49 \%$ from $30 \%$. The reduced compound $\mathbf{1} \mathbf{j}-\mathbf{H}_{\mathbf{2}}$ is an analog of the vasodilator cyclandelate. ${ }^{35}$ Given the likely role of surface interactions between electrode and substrate, it might be expected that a sterically encumbered substrate would facilitate increased recovery. In comparison, mass recoveries for benzoxazinones $\mathbf{2 a - g}$ were nearly uniformly high ( $\geq 89 \%)$. We attribute this to potentially less favourable interactions between the hydrogenated benzoxazinones and the electrode, promoting product recovery by easier dissociation. No hydrogenated product could be detected using the alkylsubstituted substrate $\mathbf{1 k}$. Attempts to replace the $\mathrm{LiClO}_{4}$ electrolyte with $\mathrm{LiCl}$ was hampered by partitioning of the water $/ \mathrm{CH}_{3} \mathrm{CN}$ solvent medium, while use of the more benign electrolyte $n \mathrm{Bu}_{4} \mathrm{NPF}_{6}$ prevented product isolation by simple extraction due to similar solubilities of electrolyte and product. Lowering the concentration of $\mathrm{LiClO}_{4}$ led to lower conversion.

From these results, a mechanism for the electrochemical hydrogenation of $\mathbf{1} \mathbf{a}-\mathbf{j}$ and $\mathbf{2 a - g}$ can be proposed. As for the electrochemical hydrogenation of aromatic $N$-heterocycles such as pyridine ${ }^{22,23}$ or phenanthridine, ${ }^{26}$ hydrogenation of $\mathbf{1 a - j}$ and 2a-g likely occurs through a surface-coupled mechanism involving reduction of protonated substrate at the electrode surface. At this point, three pathways are then feasible: (i) $\mathrm{H}_{\text {ads }}$ may be formed along with surface-adsorbed substrate, which is then subsequently be reduced by $\mathrm{H}_{\text {ads }}$ via proton-coupled electron transfer (PCET), as proposed for pyridine; ${ }^{37}$ (ii) a surface-adsorbed radical anion of protonated substrate may be further reduced to surface-adsorbed hydrogenated product, which subsequently dissociates; ${ }^{36}$ or (iii) solution-based protonated substrate may be directly reduced by $\mathrm{H}_{\text {ads }}$ to form solution-phase hydrogenated product in a $2 \mathrm{e}^{-} / 1 \mathrm{H}^{+}$process. We favour path (i) or (ii) which involve a substrate-electrode interaction, as no conversion to product is observed when using a Pt mesh working electrode, despite the proclivity of Pt metal to generate $\mathrm{H}_{\text {ads }}$ from the reduction of Bronsted acids. ${ }^{29,33}$ This interaction may be through the Lewis basic heteroatom ${ }^{38}$ or via the $\pi$-system of the substrate. ${ }^{39}$ The solid-state structures of $2 a-$ b (see $\mathrm{SI}$ ) and $\mathbf{2 g}$ (Figure 5) show a highly variable angle between the planes formed by the benzoxazine core and the aryl substituents $\left(8-43^{\circ}\right)$ which suggests a low barrier to rotation into a flattened orientation disposed to adsorb to the electrode surface. Due to the increased proton content from the three equivalents of Brønsted acid in solution, hydrogenation is more favourable than dimerization for $\mathbf{1 a - j} / \mathbf{2 a - g}$. The direct attack of an electrode-based $\mathrm{H}_{\text {ads }}$ is reminiscent of direct radical addition to unsaturated substrates. ${ }^{40}$

\section{Conclusions}

In conclusion, we present a methodology for the selective electrochemical hydrogenation of a range of aryl-substituted $\alpha$ ketoesters and benzoxazinone $\alpha$-imino-esters under electrochemically reducing conditions using carbon electrodes and a simple Brønsted acid, with high selectivity for chemoselectively hydrogenated products. Compared with existing methods for the hydrogenation of these types of substrates, no hydrogen gas, additional catalysts, redox mediators, or expensive/toxic electrode materials are required. Given the strong indications of a critical role for surface interactions, investigations into the asymmetric hydrogenation of related substrates using carbon electrodes is presently underway.

\section{Conflicts of interest}

There are no conflicts to declare.

\section{Acknowledgements}

We are grateful to the Natural Sciences Engineering Research Council of Canada for a Discovery Grant (RGPIN-2014-03733), the Canadian Foundation for Innovation and Research Manitoba for an award in support of an X-ray diffractometer (CFI \#32146), and the University of Manitoba for a USRA (BKS) and GETS support (DBN, PKG, JDB). Oscar Sze is thanked for help with preliminary experiments and Professor Rebecca L. Davis and Jorge de Carvalho Marques Dourado are thanked for SFCUHPLC chromatography.

\section{References}

1 M. Yan, Y. Kawamata and P. S. Baran, Synthetic Organic Electrochemical Methods Since 2000: On the Verge of a Renaissance, Chem. Rev., 2017, 117, 13230-13319.

2 S. Moehle, M. Zirbes, E. Rodrigo, T. Gieshoff, A. Wiebe and S. R. Waldvogel, Modern Electrochemical Aspects for the Synthesis of Value-Added Organic Products., Angew. Chem., Int. Ed., 2018, 57, 6018-6041.

3 M. Yan, Y. Kawamata and P. S. Baran, Synthetic Organic Electrochemistry: Calling All Engineers., Angew. Chem., Int. Ed., 2018, 57, 4149-4155.

4 D. S. P. Cardoso, B. Šljukić, D. M. F. Santos and C. A. C. Sequeira, Organic Electrosynthesis: From Laboratorial Practice to Industrial Applications, Org. Proc. Res. Dev., 2017, 21, 12131226.

5 J. M. Savéant and S. K. Binh, Electrochemical Hydrogenation, J. Electroanal. Chem. Interfacial Electrochem., 1974, 50, 417-419.

6 B. A. Frontana-Uribe, R. D. Little, J. G. Ibanez, A. Palma and R. Vasquez-Medrano, Organic Electrosynthesis: a Promising Green Methodology in Organic Chemistry, Green Chem., 2010, 12, 2099-2119.

7 R. S. Sherbo, R. S. Delima, V. A. Chiykowski, B. P. MacLeod and C. P. Berlinguette, Complete electron economy by pairing electrolysis with hydrogenation., Nat. Catal., 2018, 1, 501-507. 
8 P. Chambrion, L. Roger, J. Lessard, V. Béraud, J. Mailhot and M. Thomalla, The Influence of Surfactants on the Electrocatalytic Hydrogenation of Organic Compounds in Micellar, Emulsified, and Hydroorganic Solutions at Raney Nickel Electrodes, Can. J. Chem., 1995, 73, 804-815.

9 T. R. dos Santos, P. Nilges, W. Sauter, F. Harnisch and U. Schröder, Electrochemistry for the Generation of Renewable Chemicals: Electrochemical Conversion of Levulinic Acid, RSC Adv., 2015, 5, 26634-26643.

10 W. Sauter, O. L. Bergmann and U. Schröder, Hydroxyacetone: A Glycerol-Based Platform for Electrocatalytic Hydrogenation and Hydrodeoxygenation Processes, ChemSusChem, 2017, 10, 31053110.

11 R. S. Sherbo, A. Kurimoto, C. M. Brown and C. P. Berlinguette, Efficient Electrocatalytic Hydrogenation with a Palladium Membrane Reactor., J. Am. Chem. Soc., 2019, 141, 7815-7821.

12 J. M. Friedrich, C. Ponce-de-León, G. W. Reade and F. C. Walsh, Reticulated vitreous carbon as an electrode material, $J$. Electroanal. Chem., 2004, 561, 203-217.

13 A. Deronzier, J. C. Moutet and E. Saint-Aman, Electrocatalytic Hydrogenation of Organic Compounds on Carbon Electrodes Modified by Poly(pyrrole-bis[2,2'-bipyridyl] Palladium(II) Complex) Films, J. Electroanal. Chem., 1992, 327, 147-158.

14 M. N. Jackson and Y. Surendranath, Molecular Control of Heterogeneous Electrocatalysis through Graphite Conjugation, Acc. Chem. Res., 2019, 52, 3432-3441.

15 J. D. Blakemore, A. Gupta, J. J. Warren, B. S. Brunschwig and H. B. Gray, Noncovalent Immobilization of Electrocatalysts on Carbon Electrodes for Fuel Production, J. Am. Chem. Soc., 2013, 135, 18288-18291.

16 A. P. Esteves, D. M. Goken, L. J. Klein, M. J. Medeiros and D. G. Peters, Direct Electrochemical Reduction of a Bromopropargyloxy Ester at Vitreous Carbon Cathodes in Dimethylformamide, J. Electroanal. Chem., 2003, 560, 161-168.

17 L. Mattiello and L. Rampazzo, Electrochemistry of 9,9' spirobifluorene derivatives: electrosynthesis of stereoisomeric 2,3-bis(2'-acetyl-9,9'-spirobifluoren-2-yl)butane-2, 3-diols and of 1-(2'-acetyl-9,9'-spirobifluoren-2-yl)ethanol and redox properties of polyacetylated spirobifluorenes, Electrochim. Acta, 1997, 42, 2257-2264.

18 Á. Szűcs, V. Budavári, O. Berkesi and M. Novák, Electrochemical hydrogenation of C60 fullerene films, J. Electroanal. Chem., 2003, 548, 131-137.

19 J. A. Cleary, M. S. Mubarak, K. L. Vieira, M. R. Anderson and D. G. Peters, Electrochemical reduction of alkyl halides at vitreous carbon cathodes in dimethylformamide, J. Electroanal. Chem. Interfacial Electrochem., 1986, 198, 107-124.

20 J. P. Merz, B. C. Gamoke, M. P. Foley, K. Raghavachari and D. G. Peters, Electrochemical reduction of $(1 R, 2 r, 3 S, 4 R, 5 r, 6 S)$ hexachlorocyclohexane (Lindane) at carbon cathodes in dimethylformamide., J. Electroanal. Chem., 2011, 660, 121-126.

21 M. S. Mubarak and D. G. Peters, Electrochemical reduction of 1,8-dibromo- and 1,8-diiodooctane and of 1,10-dibromo- and 1,10-diiododecane at carbon cathodes in dimethylformamide., J. Electrochem. Soc., 1996, 143, 3833-3838.

22 P.-Y. Olu, Q. Li and K. Krischer, The True Fate of Pyridinium in the Reportedly Pyridinium-Catalyzed Carbon Dioxide Electroreduction on Platinum., Angew. Chem., Int. Ed., 2018, 57, 14769-14772.

23 C. X. Kronawitter, Z. Chen, P. Zhao, X. Yang and B. E. Koel, Electrocatalytic hydrogenation of pyridinium enabled by surface proton transfer reactions, Cat. Sci. Tech., 2017, 7, 831-837.
24 M. C. Groenenboom, K. Saravanan, Y. Zhu, J. M. Carr, A. Marjolin, G. G. Faura, E. C. Yu, R. N. Dominey and J. A. Keith, Structural and Substituent Group Effects on Multielectron Standard Reduction Potentials of Aromatic N-Heterocycles, J. Phys. Chem. A, 2016, 120, 6888-6894.

25 C.-H. Lim, A. M. Holder, J. T. Hynes and C. B. Musgrave, Reduction of $\mathrm{CO} 2$ to Methanol Catalyzed by a Biomimetic Organo-Hydride Produced from Pyridine, J. Am. Chem. Soc., 2014, 136, 16081-16095.

26 P. K. Giesbrecht, D. B. Nemez and D. E. Herbert, Electrochemical hydrogenation of a benzannulated pyridine to a dihydropyridine in acidic solution, Chem. Commun., 2018, 54, 338-341.

27 Q.-A. Chen, K. Gao, Y. Duan, Z.-S. Ye, L. Shi, Y. Yang and Y.-G. Zhou, Dihydrophenanthridine: A New and Easily Regenerable NAD(P)H Model for Biomimetic Asymmetric Hydrogenation, $J$. Am. Chem. Soc., 2012, 134, 2442-2448.

28 L.-Q. Lu, Y. Li, K. Junge and M. Beller, Iron-Catalyzed Hydrogenation for the In Situ Regeneration of an NAD(P)H Model: Biomimetic Reduction of $\alpha$-Keto-/ $\alpha$-Iminoesters, Angew. Chem., Int. Ed., 2013, 52, 8382-8386.

29 C. Costentin, J. C. Canales, B. Haddou and J.-M. Savéant, Electrochemistry of Acids on Platinum. Application to the Reduction of Carbon Dioxide in the Presence of Pyridinium Ion in Water, J. Am. Chem. Soc., 2013, 135, 17671-17674.

30 B. D. McCarthy, D. J. Martin, E. S. Rountree, A. C. Ullman and J. L. Dempsey, Electrochemical Reduction of Brønsted Acids by Glassy Carbon in Acetonitrile-Implications for Electrocatalytic Hydrogen Evolution, Inorg. Chem., 2014, 53, 8350-8361.

31 A. G. Wills, D. L. Poole, C. M. Alder and M. Reid, A Mechanistic and Cautionary Case Study on the Use of Alternating Potential in Electrochemical Reactions, ChemElectroChem, 2020, 7, 27712776.

32 F. Teplý, M. Čižková, P. Slavíček, V. Kolivoška, J. Tarábek, M. Hromadová and L. Pospíšil, Electron Transfer Triggers Fast Dimer/Monomer Switching of Pyridinium and Quinolinium Cations, J. Phys. Chem. C, 2012, 116, 3779-3786.

33 Y. Yan, E. L. Zeitler, J. Gu, Y. Hu and A. B. Bocarsly, Electrochemistry of Aqueous Pyridinium: Exploration of a Key Aspect of Electrocatalytic Reduction of $\mathrm{CO} 2$ to Methanol, J. Am. Chem. Soc., 2013, 135, 14020-14023.

34 D. M. Heard and A. J. J. Lennox, Electrode Materials in Modern Organic Electrochemistry, Angew. Chem., Int. Ed., 2020, 59, 18866-18884.

35 F. Heffron, B. Middleton and D. A. White, Inhibition of acyl coenzyme a: Cholesterol acyl transferase by trimethylcyclohexanylmandelate (cyclandelate), Biochem. Pharmacol., 1990, 39, 575-580.

36 M. Lessio, T. P. Senftle and E. A. Carter, Is the Surface Playing a Role during Pyridine-Catalyzed $\mathrm{CO} 2$ Reduction on $\mathrm{p}-\mathrm{GaP}$ Photoelectrodes?., ACS Energy Lett., 2016, 1, 464-468.

37 J. A. Keith and E. A. Carter, Electrochemical Reactivities of Pyridinium in Solution: Consequences for $\mathrm{CO} 2$ Reduction Mechanisms, Chem. Sci., 2013, 4, 1490-1496.

38 P. K. Giesbrecht and D. E. Herbert, Electrochemical Reduction of Carbon Dioxide to Methanol in the Presence of Benzannulated Dihydropyridine Additives., ACS Energy Lett., 2017, 2, 549-555.

39 G. Shul, M. Weissmann and D. Bélanger, Electrochemical Formation of an Ultrathin Electroactive Film from 1,10Phenanthroline on a Glassy Carbon Electrode in Acidic Electrolyte, Langmuir, 2014, 30, 6612-6621.

40 H. Miyabe, Y. Yamaoka and Y. Takemoto, Reactive Ketimino Radical Acceptors: Intermolecular Alkyl Radical Addition to 
Imines with a Phenolic Hydroxyl Group, J. Org. Chem., 2006, 71,

2099-2106. 